\title{
Wide Band Tunable Filter Design Implemented in CMOS
}

\author{
W. Matthew Anderson and Bogdan M. Wilamowski \\ Electrical \& Computer Engineering Dept. \\ Auburn University, AL 36849 \\ anderwmeauburn.edu, wilambmeauburn.edu
}

\author{
Günhan Dündar \\ Electrical and Electronic Engineering \\ Bogazici University \\ Istanbul, Turkey \\ dundar@boun. edu.tr
}

\begin{abstract}
Generation of filters using operational transconductance amplifiers and capacitors (OTA-C) has become more and more popular over recent years. A simple yet effective method of analytical synthesis is presented that makes use of ladder circuits, which are much more widely understood. Given the ladder circuit of a filter of any order, an equivalent OTA-C filter can be easily synthesized. The synthesis process is shown for both a low pass and a band pass filter. Higher order filters can easily be synthesized by extending the design process described. The design of the band pass filter is implemented in CMOS with a linearly tunable OTA to show the effect of non-ideal elements. The CMOS filter implementation is capable of linear center frequency tuning across three degrees of magnitude. Because of the wide band width tunability, this type of design lends itself to intelligent frequency scanning techniques. Such a system, to be used in military applications, is presented as an application of this filter design.
\end{abstract}

\section{INTRODUCTION}

The design of a passive ladder filter circuit based on standard filter specifications is well documented [1]. The design process is straightforward, and as such, software also exists that will calculate the necessary values of circuit elements based on given design specifications [2]. The simplicity of the ladder network provides a springboard to more complicated filter designs. This paper shows the process of transforming any ladder type circuit into an equivalent circuit containing only operational transconductance amplifiers (OTA's) and capacitors. In Section II a low pass filter (LPF) is analyzed and synthesized into an OTA-C circuit. Section III transforms the low pass prototype from Section II to a band pass filter and the new circuit is synthesized into its OTA-C equivalent. Section IV discusses the CMOS implementation of this circuit topology and shows simulations conducted with non ideal elements. An OTA is presented that is linearly tunable by varying the reference current. This OTA is used to create a band pass filter that is tunable across a wide range of frequencies. The ability to tune a filter across a very wide frequency range is advantageous in many intelligent radio systems used in the military as well as commercially. A concept for a high speed frequency scanner will be shown that is capable of using a neural network to interpret the traffic on a system.

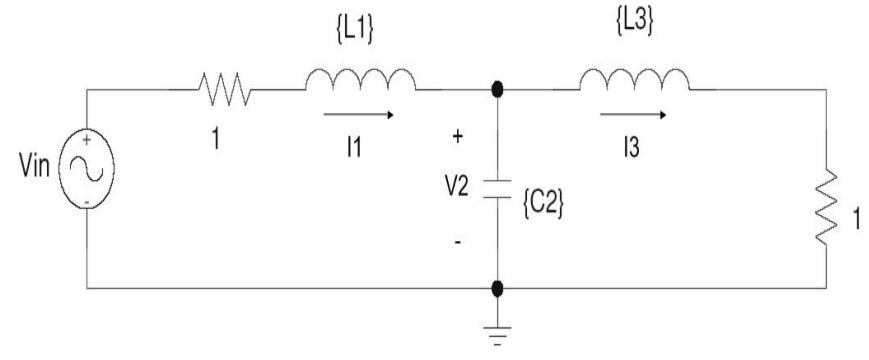

Fig. 1. Low pass filter LC implementation

\section{LOW PASS FILTER SYNTHESIS}

\section{A. LC Ladder Circuit}

The implementation of a third order Chebyshev low pass filter is sufficient to show the design process. The ladder circuit for such a filter, designed for minimum capacitance, is shown in figure 1. For simplicity, values for the passive elements are chosen to set the cut-off frequency at $1 \mathrm{~Hz}$. The frequency response of such a circuit is shown in figure 2 .

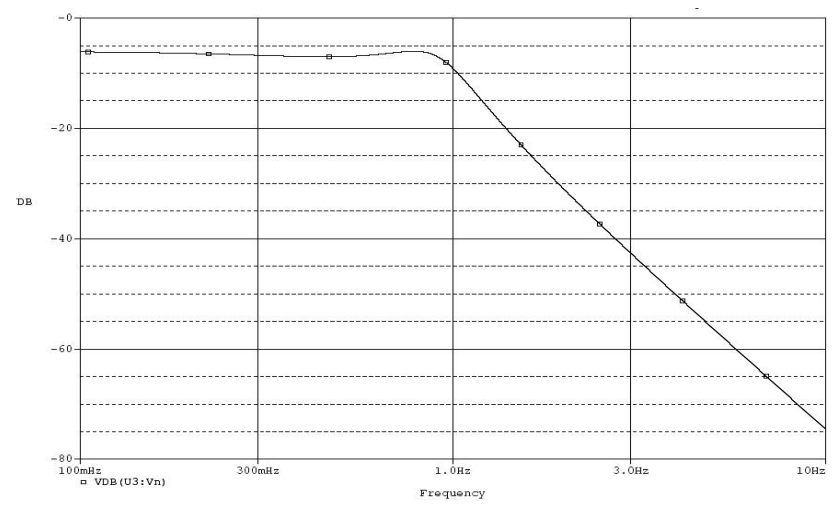

Fig. 2. Frequency response of $3^{\text {rd }}$ order prototype Chebyshev LPF

\section{B. OTA-C Transformation}

Using the current and voltage conventions defined in Figure 1 , one can easily write the state space equations for this particular circuit. 


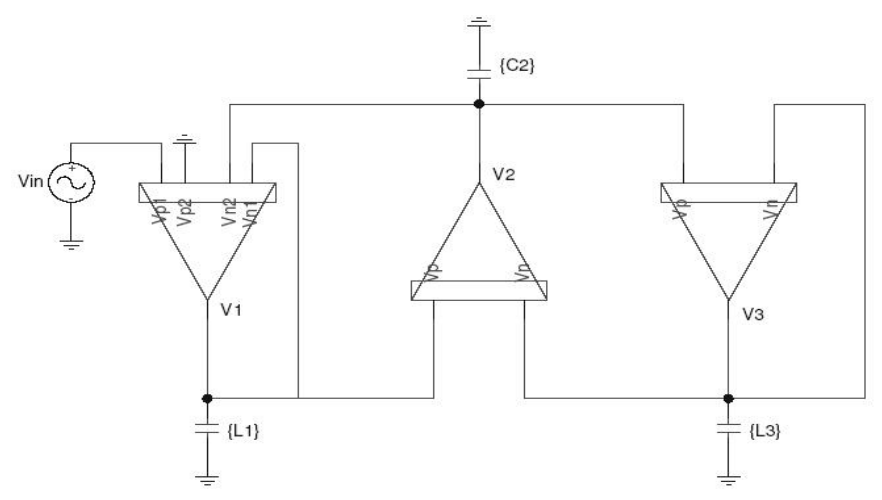

Fig. 3. Low pass filter OTA implementation

$$
\begin{aligned}
I_{1} & =\frac{1}{s L_{1}}\left(V_{\text {in }}-R_{\text {in }} I_{1}-V_{2}\right) \\
V_{2} & =\frac{1}{s C_{2}}\left(I_{1}-I_{3}\right) \\
I_{3} & =\frac{1}{s L_{3}}\left(V_{2}-R_{\text {out }} I_{3}\right)
\end{aligned}
$$

Using values of $1 \Omega$ for $R_{\text {in }}$ and $R_{\text {out }}$ and modifying the equations to use the OTA's in voltage mode, the state equations become those shown in equation set 2 .

$$
\begin{aligned}
V_{1} & =\frac{1}{s L_{1}}\left(V_{i n}-V_{1}-V_{2}\right) \\
V_{2} & =\frac{1}{s C_{2}}\left(V_{1}-V_{3}\right) \\
V_{3} & =\frac{1}{s L_{3}}\left(V_{2}-V_{3}\right)
\end{aligned}
$$

The equations in equation set 2 can be directly used to implement an active OTA-C filter in which the capacitors are all grounded [4]. However, $V_{1}$ requires an OTA with at least three inputs. The most common solution to this is to use a four input OTA and ground the fourth input. Although, if space is a concern, literature exists on a three input OTA implementation that will reduce transistor requirements [5]. Figure 3 shows the OTA-C circuit using a four input OTA where necessary. Using ideal components in SPICE, the frequency response of the circuit is exactly the same as the LC ladder response shown in figure 2. This is to be expected since, at least for the ideal case, the two circuits are equivalent.

\section{BAND PASS FILTER SYNTHESIS}

\section{A. LC Ladder Circuit}

Using the standard ladder circuit filter transformations, it is easy to determine the bandpass filter corresponding to the low pass filter from the previous section. This is the circuit shown in figure 4. As before, the values of the elements have been chosen to obtain a center frequency of $1 \mathrm{~Hz}$. The frequency response for this filter is shown in figure 5 .

\section{B. OTA-C Transformation}

One can write the circuit equations as before, using the conventions from figure 4 . These results are shown in equation set 3 . Changing the currents to voltages yields the equation set 4.

$$
\begin{aligned}
& I_{1}=\frac{1}{s L_{1}}\left(V_{i n}-I_{1} R_{i n}-V_{2}-V_{4}\right) \\
& V_{2}=\frac{1}{s C_{1}}\left(I_{1}\right) \\
& I_{3}=\frac{1}{s L_{2}}\left(V_{4}\right) \\
& V_{4}=\frac{1}{s C_{2}}\left(I_{1}-I_{3}-I_{5}\right) \\
& I_{5}=\frac{1}{s L_{3}}\left(V_{4}-V_{6}-I_{5} R_{\text {out }}\right) \\
& V_{6}=\frac{1}{s C_{3}}\left(I_{5}\right) \\
& V_{1}=\frac{1}{s L_{1}}\left(V_{i n}-V_{1}-V_{2}-V_{4}\right) \\
& V_{2}=\frac{1}{s C_{1}}\left(V_{1}\right) \\
& V_{3}=\frac{1}{s L_{2}}\left(V_{4}\right) \\
& V_{4}=\frac{1}{s C_{2}}\left(V_{1}-V_{3}-V_{5}\right) \\
& V_{5}=\frac{1}{s L_{3}}\left(V_{4}-V_{6}-V_{5}\right) \\
& V_{6}=\frac{1}{s C_{3}}\left(V_{5}\right)
\end{aligned}
$$

However, this is a slightly more complicated case. The difficulty arises from the equation for $V_{1}$. Although one can easily fabricate a four input OTA, it will obviously have two positive inputs and two negative inputs. Using the equations from equation 4 will result in the OTA for $V_{1}$ needing three negative inputs. To remedy this situation, it is necessary to manipulate the signs of the variables. It can be clearly seen that if $V_{2}$ were positive in the equation for $V_{1}$ then a standard

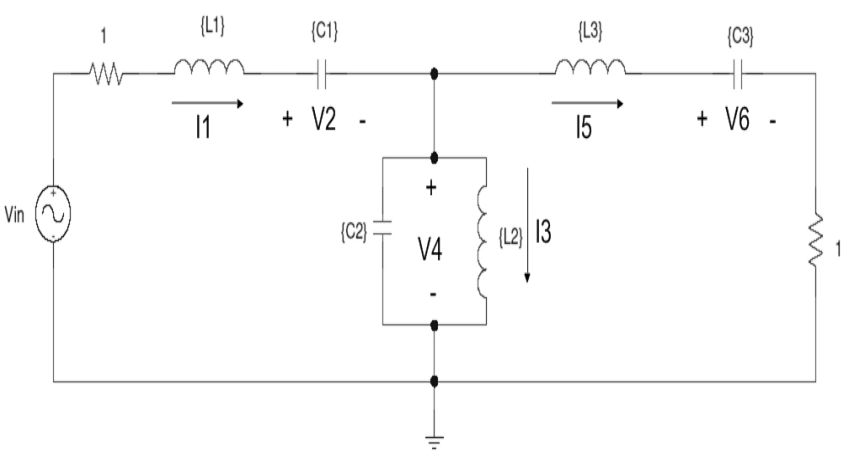

Fig. 4. Band pass filter ladder circuit 


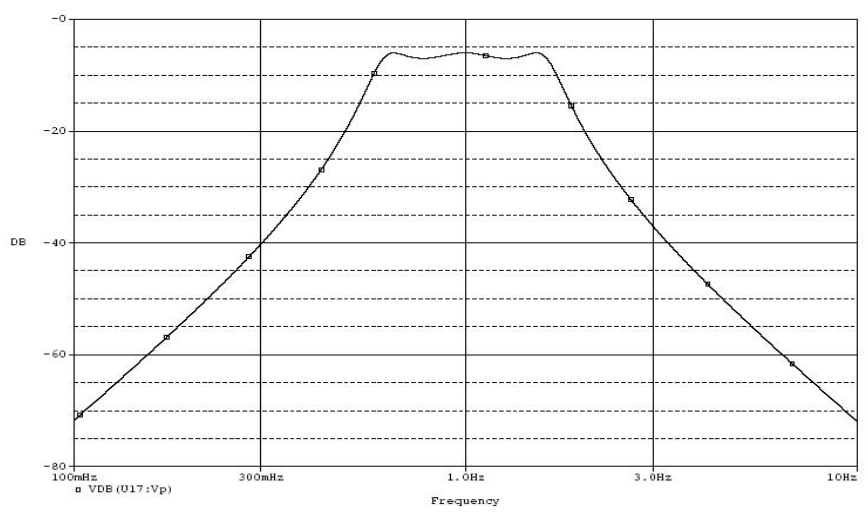

Fig. 5. Frequency response of $6^{\text {th }}$ order prototype Chebyshev BPF

four input OTA could be used in the circuit. This being the case, changing the sign of $V_{2}$ everywhere it occurs in equation set 4 results in equation set 5 .

$$
\begin{aligned}
V_{1} & =\frac{1}{s L_{1}}\left(V_{\text {in }}-V_{1}+V_{2}-V_{4}\right) \\
V_{2} & =\frac{1}{s C_{1}}\left(-V_{1}\right) \\
V_{3} & =\frac{1}{s L_{2}}\left(V_{4}\right) \\
V_{4} & =\frac{1}{s C_{2}}\left(V_{1}-V_{3}-V_{5}\right) \\
V_{5} & =\frac{1}{s L_{3}}\left(V_{4}-V_{6}-V_{5}\right) \\
V_{6} & =\frac{1}{s C_{3}}\left(V_{5}\right)
\end{aligned}
$$

The idealized circuit corresponding to equation set 5 is shown in figure 6. As in the case of the low pass circuit, the filter response is exactly the same as show in figure 5 .

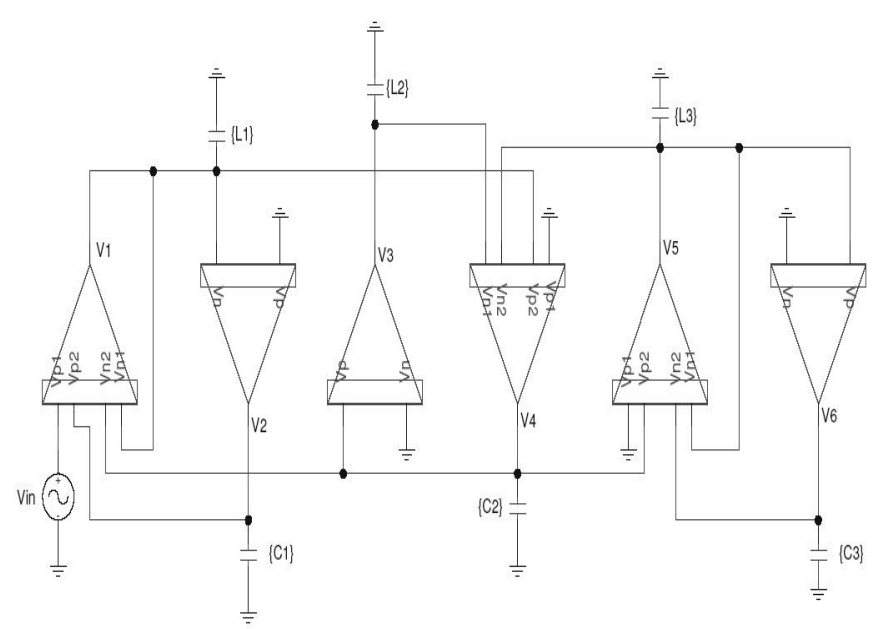

Fig. 6. Band pass filter OTA implementation

\section{CMOS IMPLEMENTATION}

It is desirable, especially in the case of a band pass filter, to be able to linearly tune the center frequency of the filter. In the case of the previously discussed OTA-C designs, this means transconductance $\left(g_{m}\right)$ must be linearly tunable. Examples of linearly tunable OTA's can be found in the literature [3], [6], [7]. For the implementation that follows, an OTA is designed that is linearly tuned by the reference current supplied to the OTA. This technique is preferred because a single current source can be routed to all OTA's in a filter. To tune the filter center frequency, only one current source need be adjusted. The OTA designed for this purpose is shown in figure 7.

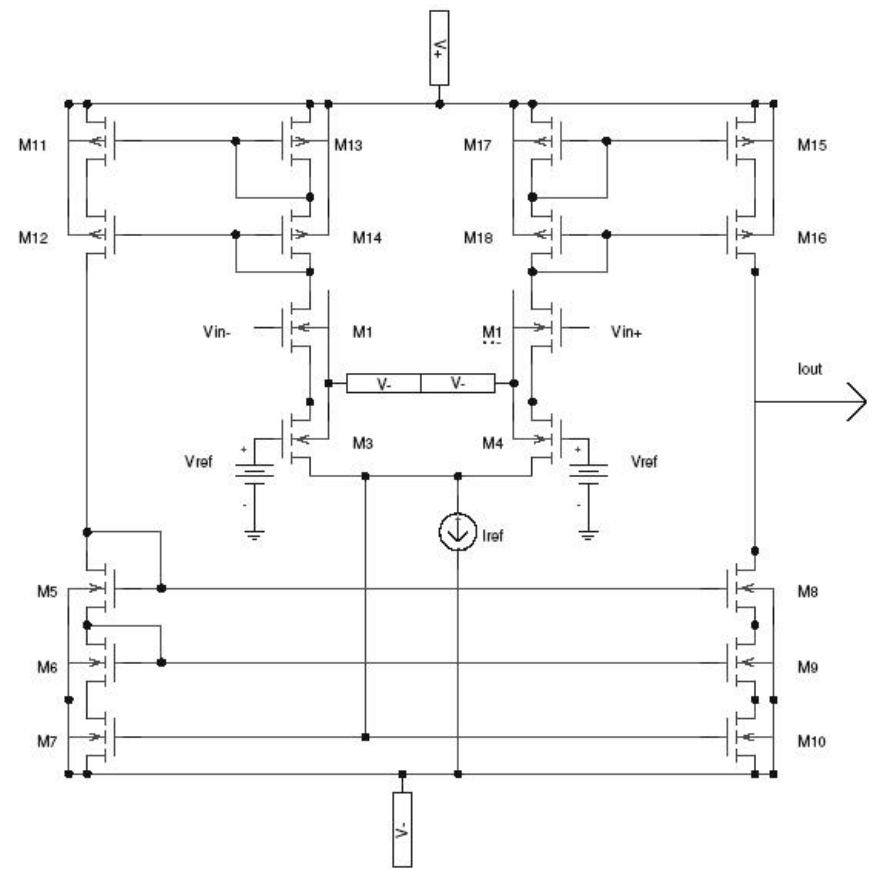

Fig. 7. Linear current tunable OTA

It is not a trivial matter to design an OTA that is suited to be used in the previously designed filter structures. It is necessary for the device to have a large transconductance parameter and a large output resistance. This is most easily accomplished by designing an amplifier that has relatively high transconductance at small quiescent currents. Transistor pair M3 and M4 are used to increase the linearity of the device. The drawback of this design is the requirement of a precise voltage reference on the gates of these transistors to ensure proper operation. The choice of this voltage represents a trade off between linearity and gain. Because of this, the characteristics of a filter using this particular OTA are heavily dependent on the $V_{\text {ref }}$ voltage. On the other hand, there are several advantages gained by this circuitry. Most importantly, transconductance remains linear for over four degrees of magnitude. Figure 8 shows how the linearity of transconductance is affected by the biasing current. The corollary of this is shown in figure 9. Since the transconductance is linear with reference current, the output resistance of the device is linear as well. When the 


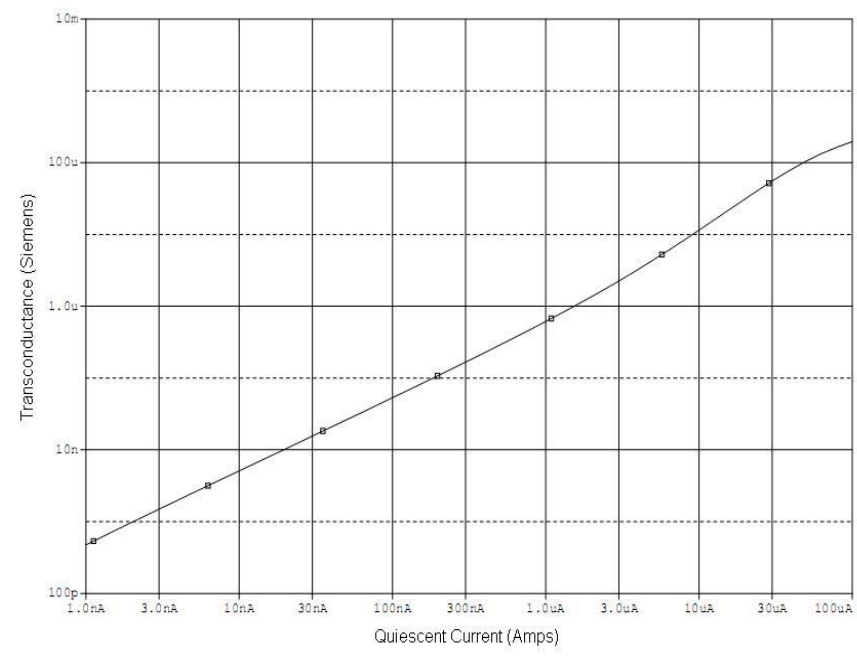

Fig. 8. Transconductance verses quiescent current

output resistance gets sufficiently small (around $300 \mathrm{k} \Omega$ ), the filter shape begins to degrade. This effect limits the filter at larger values of current.

To show how the OTA impacts the circuit, the ideal OTA's in the band pass filter discussed in Section III will be replaced with the OTA circuit of figure 7. The simulation results in Figure 10 shows the frequency response of the band pass filter of Section III with the non-ideal OTA. The transistor models used in the simulation are taken from the AMIS $0.5 \mu \mathrm{m}$ process. It should be noted that the quality factor of the filter is set externally by the choice of capacitor values. However, the center frequency of the filter is determined by the transconductance of the operational transconductance amplifier. The transconductance is, in the case of the amplifier shown in figure 7 , linearly related to the supply current. The four plots in the figure represent the reference current being

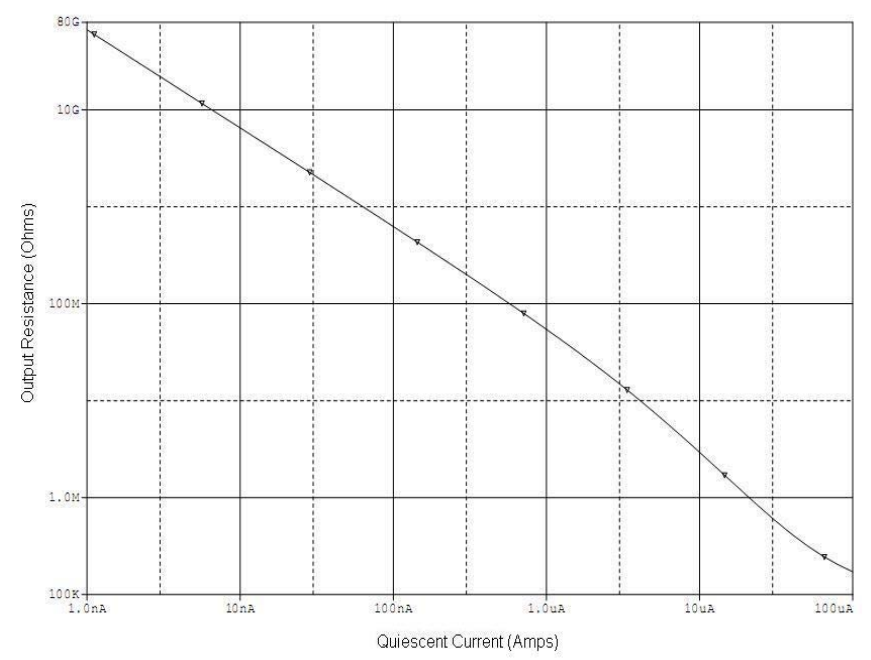

Fig. 9. Output resistance verses quiescent current

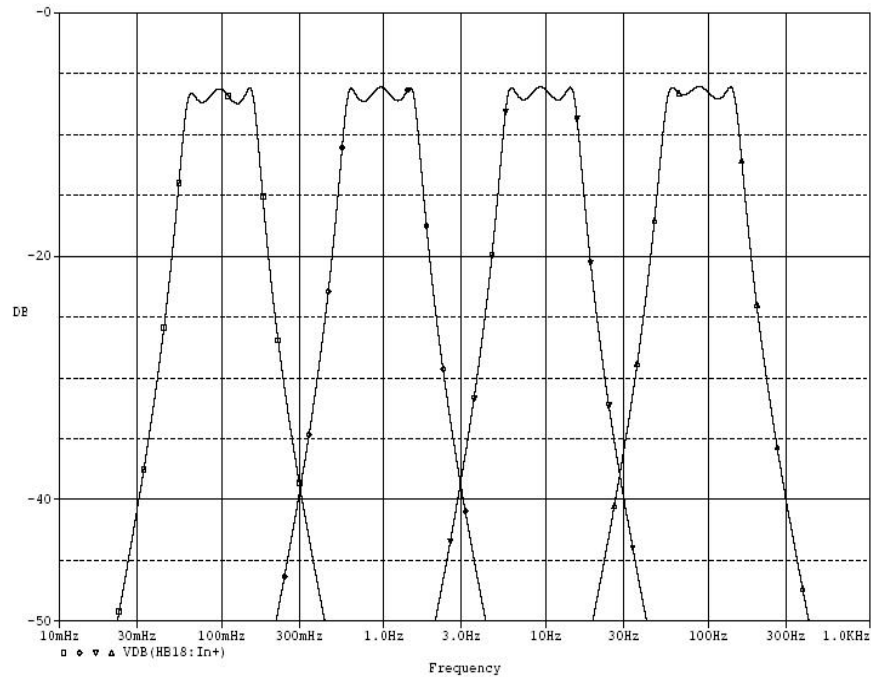

Fig. 10. Prototype band pass filter implemented with tunable OTA. The curves represent $I_{\text {ref }}$ values of $10 \mathrm{nA}, 100 \mathrm{nA}, 1 \mu \mathrm{A}$, and $10 \mu \mathrm{A}$ respectively

stepped from $10 \mathrm{nA}$ to $10 \mu \mathrm{A}$ by the decade. As can be seen from figure 10 , an increase of a degree of magnitude in reference current results in an increase in a degree of magnitude of the filter center frequency.

For a more practical example, figure 11 shows how the band pass filter responds at higher frequencies. This is accomplished by scaling down all of the capacitor values in the prototype by a constant value. To obtain the frequencies shown in figure 11 the capacitor values are on the order of $10 \mathrm{pF}$. The band pass filter with those capacitor values can be tuned from $100 \mathrm{~Hz}$ to $100 \mathrm{KHz}$ while operating on a reference current from $10 \mathrm{nA}$ to $10 \mu \mathrm{A}$. Figure 12 shows how the filter operates

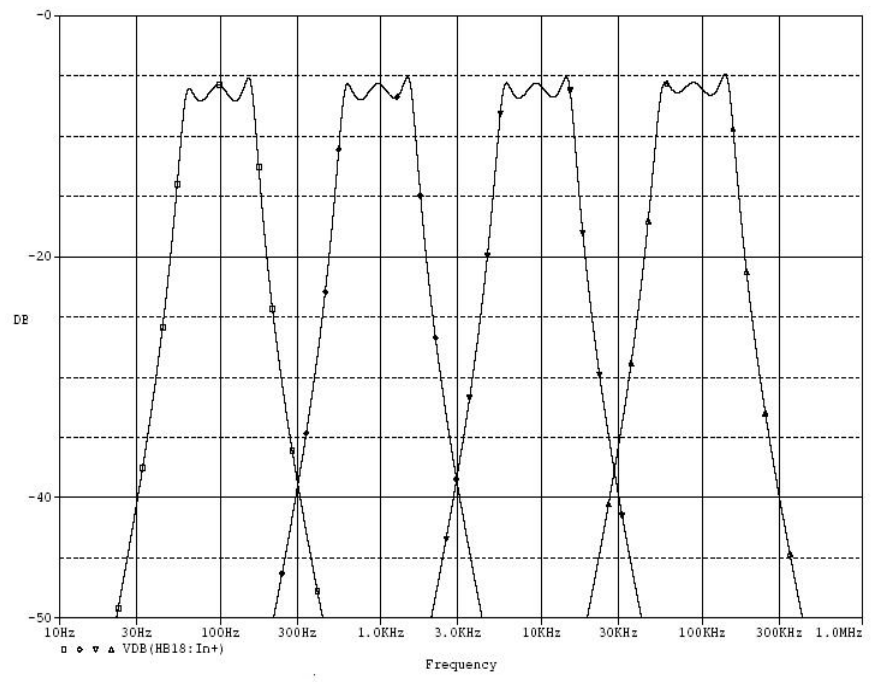

Fig. 11. Tunable band pass filter operating at higher frequencies. The curves represent $I_{\text {ref }}$ values of $10 \mathrm{nA}, 100 \mathrm{nA}, 1 \mu \mathrm{A}$, and $10 \mu \mathrm{A}$ respectively

at higher quality factors. The filter response becomes limited by the performance of the OTA. The performance can be 
altered by adjusting the reference voltage $V_{\text {ref }}$ shown in figure 7 but some linearity will be sacrificed for the increase in transconductance. The trade off between transconductance and linearity is the major design decision when using this OTA implementation.

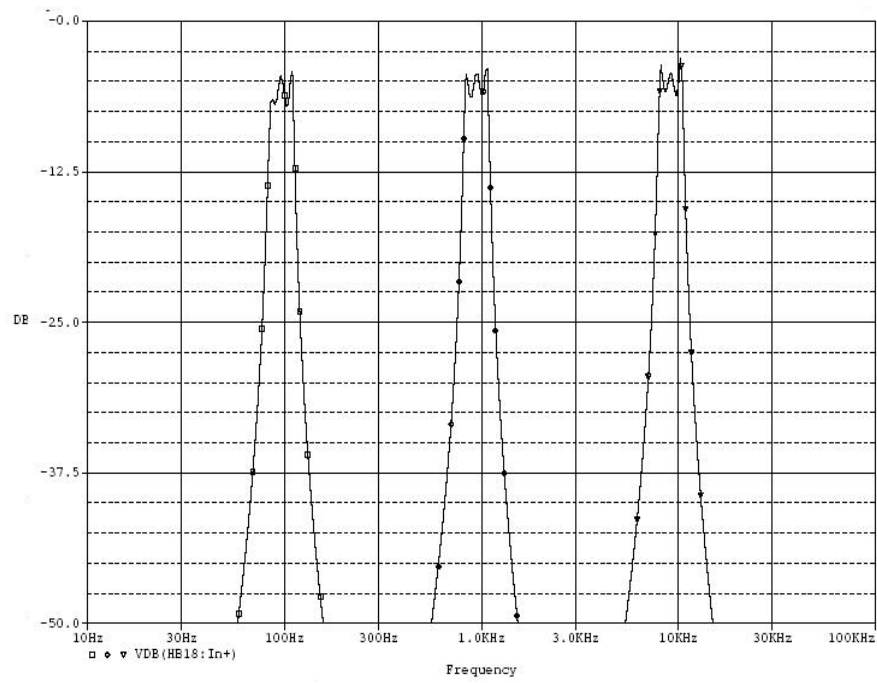

Fig. 12. Tunable band pass filter operating at higher frequencies and higher $\mathrm{Q}$. The curves represent $I_{\text {ref }}$ values of $10 \mathrm{nA}, 100 \mathrm{nA}$, and $1 \mu \mathrm{A}$ respectively

The applications of such a filter are far reaching. One practical example is in the design of a high speed frequency scanner. Frequency scanners find use in several military applications. For one, they can be used in conjunction with a radio jammer to thwart explosives that use a cell phone or other radio device as the detonator. As another example, in times of war a sudden increase in radio traffic often corresponds to a coming enemy attack. In such a device, it is advantageous to be able to scan large ranges of frequencies at very high speeds. The previously shown current tunable band pass filter is well equipped to perform such a task. It performs well for three decades of center frequencies and the scan can be conducted as fast as the current source can be varied. Due to the speed in which the filter can scan, a sophisticated method of interpreting the output of the filter must be determined. The output will be in the form of pulses of information whenever the scanning filter rolls over a frequency that is transmitting. Given these pulses as an input, along with the normalized reference current, a neural network can be trained that will warn of an impending attack.

Continued research is needed in several areas. For one, the OTA shown in figure 7 can be improved upon. An improvement in slew could facilitate filters with higher higher quality factors. Also, higher center frequencies could be reached if the bandwidth of the OTA were improved. The concept of an intelligent radio scanner is in its early stages of research but shows considerable promise. Using a neural network to interpret the traffic it will be possible to detect wartime radio patterns much more efficiently. This, in turn, will increase the warning time preceding an enemy attack.

\section{CONCLUSION}

A concise method of synthesizing OTA-C filters from ladder circuits has been presented. This method is effective for any ladder circuit, but in some cases requires multiple (more than two) input OTA's. A linearly tunable OTA was shown that can be used in the implementation of OTA-C filters. The OTA provides a trade-off between transconductance and linearity in the form of a reference voltage. A band pass filter was synthesized and implemented using this OTA that achieves mostly linear tuning across three degrees of magnitude. The limitations of the OTA were shown, and more research is to be done on improving on the OTA design. The concept of a intelligent radio scanner was developed that uses the attributes of the band pass filter in conjunction with a neural network to warn military personnel of suspicious radio traffic. Other applications are readily available for such a versatile filter structure.

\section{Acknowledgments}

This work was supported in part by NSF international grant US-Turkey Cooperative Research: Silicon implementation of computational intelligence for mechatronics. NSF OISE 0352771

\section{REFERENCES}

[1] R. D. Koller and B. M. Wilamowski, "A ladder Prototype Sythesis Algorithm,", 35th Midwest Symp. Circuits and Systems, Washington, DC, USA, Aug. 9-12, 1992.

[2] Robert D. Koller and Bogdan Wilamowski, "LADDER-A Microcomputer Tool for Passive Filter Design and Simulation," IEEE Transactions on Education, vol. 39, no. 4, Nov. 1996.

[3] A. Nedungad And T. R. Viswanathan, "Design of Linear CMOS Transconductance Elements," IEEE Transactions on Circuits and Systems, vol. cas-31, no. 10, Oct. 1984.

[4] Yichuang Sun, "Synthesis of Leap-Frog Multiple-Loop Feedback OTA-C Filters," IEEE Transactions on Circuits and Systems Express Briefs, vol. 53, no. 9, Sept. 2006.

[5] M. Jeong, S. Takagi, Z. Czarnul, and N. Fugii "Design Of a Novel Linear 3-Input CMOS OTA and Its Application to Filter Realization," Proceedings of IEEE Asia Pacific Conference on Circuits and Systems, Seoul, Korea, pp. 121-124, 1996

[6] Milind Subhash Sawant, Jaime Ramrez-Angulo, Antonio. J. Lpez-Martn, and Ramon G. Carvajal "Wide gm Adjustment Range Highly Linear OTA with Programmable Mirrors Operating in Triode Mode," 2006 IEEE International Symposium on Circuits and Systems, 21-24 May 2006 Page(s):4 $\mathrm{pp}$

[7] Yunbin Deng, S. Chakrabartty, and G. Cauwenberghs "Three-decade programmable fully differential linear OTA," 2004 IEEE International Symposium on Circuits and Systems, Volume 1, 23-26 May 2004 Page(s):I - 697-700. 